\title{
The new risk variant CACNA1C and brain circuits in schizophrenia
}

\author{
Andrea Schmitt • Peter Falkai
}

Published online: 31 January 2014

(c) Springer-Verlag Berlin Heidelberg 2014

In recent genome-wide association studies of severe psychiatric disorders, several risk genes have been detected, but their neurobiological function is widely unknown. A polymorphism in the gene encoding the alpha-1C subunit of the L-type voltage-gated calcium channel (CACNA1C) has been associated with schizophrenia and bipolar disorder and may have impact on structure and function of the amygdala. Wolf et al. [1] investigated the influence of the SNP rs1006737 in CACNA1C on gray matter volumes of the amygdala in patients with schizophrenia, bipolar disorder and obsessive compulsive disorder (OCD). The risk genotype had significant effect on the relative amygdala volume in patients with schizophrenia, while this group and bipolar patients had a smaller left amygdala compared to controls. This supports the hypothesis of disturbed calcium metabolism as a neurobiological background of affective symptoms in schizophrenia. However, CACNA1C is also known to be involved in learning and memory. In a functional MRI study of two independent samples of healthy probands, Krug et al. [2] investigated the impact of rs1006737 on episodic memory encoding and retrieval in the right hippocampus. In both samples, they replicated that in the retrieval condition, carriers of the minor allele (A) had lower activations than $\mathrm{G} / \mathrm{G}$ allele carriers, thus strengthening the hypothesis that this risk genotype is associated with dysfunction of hippocampus-related memory processes.

Schizophrenia has been linked to disturbances in neuronal circuits such as the cortico-striato-thalamo-cortical

A. Schmitt $(\bowtie) \cdot$ P. Falkai

Department of Psychiatry and Psychotherapy,

Ludwig-Maximilians-University Munich, Nußbaumstr. 7,

80336 Munich, Germany

e-mail: andrea.schmitt@med.uni-muenchen.de loop. As a consequence, disinhibition of the thalamus plays a prominent role in the overflow of information to the prefrontal cortex and possibly is involved in the generation of positive symptoms. In a resting-state functional MRI study, Klingner et al. [3] found higher thalamocortical functional connectivity in chronic schizophrenia patients compared to healthy controls. Specifically, the connectivity to the right ventrolateral prefrontal and bilateral motor and sensory cortical areas was affected. Structural analysis revealed involvement of medial and anterior thalamic nuclei in correlation with the prefrontal and superior temporal cortex, contributing to alterations of the network in schizophrenia. However, treatment with antipsychotics may have an influence on macroconnectivity and myelination of fiber tracts. In a cell culture study of oligodendrocytes with or without the presence of the cuprizone, a white matter damaging agent, $\mathrm{Xu}$ et al. [4] reported that clozapine and quetiapine, but not haloperidol or olanzapine counteracted effects of cuprizone. These findings are relevant for the growing knowledge about the influence of antipsychotics on pathophysiological aspects such as myelination-related dysconnectivity in schizophrenia. In a clinical assessment of 56.861 schizophrenia patients, Grohmann et al. [5] compared the typical neuroleptic flupentixol to haloperidol and second-generation antipsychotics with respect to drug utilization and adverse drug reactions (ADR). Flupentixol ranked lowest among all ADR. Extrapyramidal symptoms were most frequently detected in flupentixol and risperidone and least in the olanzapine-/quetiapine-treated group. Altogether, the results justify the treatment approach with flupentixol in a subgroup of schizophrenia patients.

Disturbances in semantic processing are proposed to be involved in the pathophysiology of formal thought disorder in schizophrenia. In a functional MRI study, Sass et al. [6] 
demonstrated effects of semantic relation within the right angular gyrus and precuneus, thus differentiating patients $>$ controls. In contrast, the effect of modality revealed differences in controls $>$ patients within the left superior frontal, middle temporal, inferior occipital, right angular gyrus and anterior cingulate cortex. The increased activity in the patient group may reflect delayed and enhanced spread of activation within semantic networks, while modality-specific decreases may be linked to impaired perceptual integration, indicating pathophysiological aspects of impaired speech processing in schizophrenia.

Schizophrenia is known to be related to increased vulnerability. In a large group of referrals for suspected psychosis, Raballo et al. [7] identified four vulnerability factors: dysphoria as irritability tension, paranoid autocentrism, introversive withdrawal and disturbed subjective experience. Latent class analysis distinguished three classes as carrying different degrees of proneness to schizophrenia psychosis and supports them as underlying continuum of clinical severity. In addition, anxiety is a common symptom in schizophrenia, but assessment is difficult due to the lack of specific evaluation tools. Llorca et al. [8] present a rating scale for the evaluation of anxiety in schizophrenia (SAES) which they validated in a large patient group. It is composed of three factors, namely "expressed end perceived anxiety," "somatic anxiety" and "anxiety and environment". The scale shows good interrater reliability and can be recommended for assessment of anxiety symptoms in schizophrenia.

According to the inflammation hypothesis, schizophrenia has been linked to infections like toxoplasma gondii. Antipsychotics and mood stabilizers have anti-toxoplasmic properties, which have been in the focus of investigation in the present in vitro study by Fond et al. [9]. They found that fluphenazine has high anti-toxoplasmic activity, but this effect is also shared by zuclopenthixol. In contrast, valproate, tiapride and amisulpride have no impact on parasite growth, and other antipsychotics show only low activity. However, concentration of these drugs in the brain are unknown in humans, and therefore, it is unknown, which effects these drugs have in Toxoplasma-positive patients. More research of the influence of anti-inflammatory drugs on schizophrenia is required.

\section{References}

1. Wolf C, Mohr H, Schneider-Axmann T, Reif A, Wobrock T, Scherk H, Gruber O (2013) CACNA1C genotype explains interindividual differences in amygdala volume among patients with schizophrenia. Eur Arch Psychiatry Clin Neurosci. doi:10. 1007/s00406-013-0427-y

2. Krug A, Witt SH, Backes H, Dietsche B, Nieratschker V, Shah NJ, Kircher T (2013) A genome-wide supported variant in CACNA1C influences hippocampal activation during episodic memory encoding and retrieval. Eur Arch Psychiatry Clin Neurosci. doi:10.1007/ s00406-013-0428-x

3. Klingner CM, Kerstin L, Maren D, Stefan S, Otto WW, Heinrich S, Igor N (2013) Thalamocortical connectivity during resting state in schizophrenia. Eur Arch Psychiatry Clin Neurosci. doi:10.1007/ s00406-013-0417-0

4. Xu H, Hong-Ju Y, Xin-Min L (2013) Differential effects of antipsychotics on the development of rat oligodendrocyte precursor cells exposed to cuprizone. Eur Arch Psychiatry Clin Neurosci. doi:10.1007/s00406-013-0414-3

5. Grohmann RR, Engel R, Möller H-J, Rüther E, van der Velden JW, Stübner S (2013) Flupentixol use and adverse reactions in comparison with other common first-and second-generation antipsychotics: data from the AMSP study. Eur Arch Psychiatry Clin Neurosci. doi:10.1007/s00406-013-0419-y

6. Sass K, Stefan H, Olga S, Benjamin S, Frank S, Ute H, T Kircher (2013) Neural correlates of semantic associations in patients with schizophrenia. Eur Arch Psychiatry Clin Neurosci. doi:10.1007/ s00406-013-0425-0

7. Raballo A, Anna M, Angelo C, Davide S, Marco BLR, Andrea A, Maria TC, Antonio P, Kurt M, Heinz H (2013) Shades of vulnerability: latent structures of clinical caseness in prodromal and early phases of schizophrenia. Eur Arch Psychiatry Clin Neurosci. doi:10.1007/s00406-013-0421-4

8. Llorca P-M, Christophe L, Olivier B, Ingrid de C, Ludovic S, Hervé C, Jean-Alexandre L, Franck JB (2013) A composite scale applied to evaluate anxiety in schizophrenic patients (SAES). Eur Arch Psychiatry Clin Neurosci. doi:10.1007/s00406-013-0416-1

9. Fond G, Alexandra M, Ryad T, Nora H, Alexandre M, Marion L, Jean-Francois D (2013) Comparative analysis of anti-toxoplasmic activity of antipsychotic drugs and valproate. Eur Arch Psychiatry Clin Neurosci. doi:10.1007/s00406-013-0413-4 